\title{
Brotes de salmonelosis y el tamaño y rol del Estado en Chile
}

\author{
Alberto Fica, Gerardo Acosta, Jeannette Dabanch, Cecilia Perret, Marisa Torres, \\ Javier López, Leonor Jofré y Thomas Weitzel por el Comité de Infecciones Emergentes \\ de la Sociedad Chilena de Infectología
}

\section{Salmonellosis outbreaks and the size and role of the Chilean State}

During year 2011 two outbreaks of Salmonella infection captured media attention in the Metropolitan Area (MA) in Chile: one of typhoid fever associated to Salmonella serotype Typhi, and the other, of gastroenteritis related to Salmonella serotype Enteritidis, both with decreasing or stable rates in the previous years. The aim of this work is to analyze probable causes of their reemergence. Methods: Several government websites were searched looking for epidemiological data. Results: Typhoid fever rates have declined to current values of 1 case per 100.000 habitants, a decreased associated to improvements in the human development index. The typhoid outbreak was associated to a predominant clone within the MA. The only risk factor identified was consumption of raw vegetables acquired in open fairs, but without identifying a common source. Despite improvements in disease notification and molecular epidemiology capabilities, this outbreak is coincidental with a reduced number of food inspection visits in the MA, probably explained by the limited personnel available for this task. In the case of Salmonella Enteritidis, rates have increased twice since 1998 (5.3 to 10.7 per 100.000 habitants) with an important increase in the number of outbreaks linked to this agent (7 to 31 annual outbreaks) since year 2005. Persistence of this problem is probably associated to the low surveillance of poultry farms made by the Chilean state, to the absence of a cold chain during collection, distribution and selling of eggs, and to the lack of an educational program directed to the population. The recent regulation that bans home-made mayonnaise in restaurant or fast food stores is an important advance that requires further evaluation. Conclusions: The persistence and reemergence of different kind of Salmonellosis in Chile suggests chronic problems on the size and role of the Chilean state regarding food safety.

Key words: Salmonella infections, Salmonella food poisoning, typhoid fever, disease outbreaks, epidemiology, food contamination, food industry.

Palabras clave: Infecciones por Salmonella, intoxicaciones por Salmonella, fiebre tifoidea, brotes, epidemiología, contaminación de alimentos, industria de alimentos.
Hospital Militar de Santiago, Chile.

Servicio de Infectología (AF, JD). Universidad Austral, Valdivia, Chile.

Facultad de Ciencias Veterinarias Instituto de Medicina Veterinaria Preventiva (GA)

Pontificia Universidad Católica de Chile, Santiago.

Facultad de Medicina Departamento de Pediatría (CP). Departamentos de Salud Pública y Laboratorios Clínicos (MT).

Clínica Veterinaria Alcántara (JL). Hospital Clínico Universidad de Chile.

Servicio de Pediatría (ப). Clínica Alemana / Universidad del Desarrollo, Santiago, Chile (TW).

Conflicto de interés: ninguno Fuente de financiamiento: ninguna

Recibido: 10 de enero de 2012 Aceptado: 18 de enero de 2012

Correspondencia a: Alberto Fica Cubillos albertofica@gmail.com
$\mathrm{D}$ urante el año 2011 se detectaron en Chile dos brotes de infecciones asociadas al género Salmonella en la Región Metropolitana (RM), los que fueron ampliamente difundidos en diferentes medios de prensa generando alarma pública ${ }^{1,2}$. Uno de ellos, de fiebre tifoidea, asociado a Salmonella enterica serotipo Typhi (Salmonella Typhi), de reservorio exclusivamente humano, y el otro, de gastroenteritis aguda por Salmonella enterica serotipo Enteritidis (Salmonella Enteritidis), ligado principalmente a un reservorio avícola. En ambos casos de infección, el contagio se produce por ingesta de agua y/o alimentos contaminados, pero de diferente origen. Las formas de control y prevención en ambos casos difieren ya que tienen diferentes reservorios, fuentes de infección y mecanismos de transmisión. El objetivo de este trabajo es describir la situación de estas salmonelosis en Chile y explorar posibles causas asociadas a su persistencia o expansión reciente. Se analizará en forma consecutiva el problema de fiebre tifoidea y luego el de Salmonella Enteritidis.

\section{Salmonelosis, evolución en el tiempo y relación con el desarrollo humano}

Los datos de fiebre tifoidea (enfermedad de notificación obligatoria) fueron obtenidos de las páginas oficiales del Departamento de Epidemiología del Ministerio de Salud de Chile (http://epi.minsal.cl) y del Departamento de Estadísticas e Información de Salud del mismo ministerio (http://www.deis.cl). Los datos sobre casos de infección por Salmonella serotipo Enteritidis (enfermedad no notificable) y los resultados de la tipificación molecular de este agente y de Salmonella Typhi fueron extraídos de los informes recientes emanados por el Instituto de Salud Pública de Chile (ISP) presentes en la página institucional (http://www.ispch.cl/) y por el Departamento de Epidemiología del Ministerio de Salud ${ }^{3,4}$. Las tasas de 
infecciones por Salmonella Enteritidis fueron expresadas por tamaño poblacional usando estimaciones de población del Instituto Nacional de Estadísticas y comparadas con datos previos publicados hace más de una década, ${ }^{5,6}$. La correlación entre tasas de fiebre tifoidea y el índice de desarrollo humano (IDH) fue efectuada mediante el coeficiente de correlación de Pearson para ambos parámetros entre 1980 y el 2010. Los datos sobre IDH para Chile fueron rescatados desde informes del Programa de la Naciones Unidas para el Desarrollo (PNUD) ${ }^{7}$.

\section{Normativa y fiscalización alimentaria}

La normativa vigente fue obtenida desde el Reglamento Sanitario de los Alimentos, emitido el año 1996 y actualizado en junio del 2010 (DTO N977/96). Esta normativa está disponible en el sitio web del Ministerio de Salud $^{8}$. La información sobre actividades de fiscalización en alimentos efectuadas por las Secretarías Regionales Ministeriales de Salud (Seremi-Salud), fueron extraídas de su sitio web (http://www.asrm.cl). Los datos fueron analizados respecto a su variación en el tiempo y por tamaño poblacional para cada región. A su vez, los datos de tamaño poblacional, fueron obtenidos de las proyecciones de población global y por regiones desde la página web del Instituto Nacional de Estadísticas (http://www.ine.cl) ${ }^{5}$.

Los resultados del trabajo son presentados en paralelo a la discusión para permitir una mejor comprensión de la situación epidemiológica y sus potenciales causas.

\section{Fiebre tifoidea}

La tasa de incidencia de esta enfermedad ha declinado progresivamente desde los años 80 a cifras actuales de 1 caso por 100.000 habitantes (Figura 1). Al asociar la declinación a las mejorías en las condiciones de vida de la población que se expresan por el índice de desarrollo humano que ha alcanzado el país y que reflejan entre otros aspectos, mejores niveles de educación, cobertura de salud, acceso a agua potable, alcantarillado, recolección de residuos sólidos y manejo de aguas servidas, se observa una relación inversa y estadísticamente significativa ( $\mathrm{r}-0,93$, $\mathrm{p}<0,05$ por prueba de correlación de Pearson; Figura 2).

La transmisión fecal-oral del grupo de agentes del género Salmonella que explica la fiebre tifoidea (serotipos Typhi, Paratyphi A, B y C), se ve imposibilitada de alcanzar un nuevo hospedero susceptible desde su reservorio exclusivo humano, gracias a este avance sanitario, el que limita nuevos casos a distancia más allá del entorno cercano (ciclo largo). De esta manera, el mayor desarrollo humano permite tanto el control de brotes epidémicos como una menor tasa endémica de fiebre tifoidea, pero no logra impactar sobre el universo de ciclos cortos de transmisión (entorno familiar o laboral) donde portadores crónicos de Salmonella serotipos Typhi o Paratyphi que contaminan agua o alimentos a través de sus deposiciones, igual pueden producir casos esporádicos o brotes limitados.

El brote de fiebre tifoidea que captó la atención del público por diferentes medios de prensa en el período agosto-septiembre del año 2011 y que se detalla a continuación (Figura 3), de alguna manera quiebra esta curva en declinación ${ }^{3}$. El brote ocurrió entre las semanas epidemiológicas 32 y 38 en la RM, especialmente en dos áreas geográficas (Servicios de Salud Metropolitano Central y Metropolitano Occidente).

Específicamente en las cuatro comunas más afectadas en estos dos Servicios de Salud, la tasa de incidencia varió entre 1,9 y 5,8 casos por 100.000 habitantes, muy superior a la mediana nacional de los últimos años. Además, 13 de los 17 casos identificados entre las semanas 32 y 38, presentaron una cepa que pertenece al mismo genotipo según la electroforesis de campos pulsados, la que además no había sido pesquisada previamente en el país $^{3}$. Las investigaciones epidemiológicas efectuadas por la SEREMI de Salud de la RM ante este brote, ha podido identificar como factor único común el consumo de verduras adquiridas en ferias libres de la zona occidente de Santiago. Incluso dos de estos casos fueron observados en hijos de comerciantes de estas ferias libres y tres casos fueron observados en el mismo grupo familiar. En ellos, el jugo de zanahoria cruda resultó ser el alimento contaminado más probable de este brote intra-domiciliario. Por otra parte, la investigación de campo no identificó casos en inmigrantes ni en personas que consumieron verduras picadas en venta en estas ferias (Comunicación personal SEREMI Salud RM). En la Figura 4 se pueden apreciar los casos en la RM observados hasta la semana 39 del 2011 y como éstos se concentran en el sector poniente desde la semana 25 en adelante ${ }^{3}$.

Este brote de fiebre tifoidea en el momento actual que se caracteriza por bajas tasas de incidencia, genera dos interrogantes: por una parte aclarar el origen de este brote y por otra, analizar si tenemos un sistema de respuesta epidemiológica preparado para esta contingencia. A pesar del período de incubación prolongado de esta enfermedad, que bordea las dos semanas, el bajo número de casos debería permitir la identificación de la potencial fuente de origen y su control si existiera un sistema ágil de sospecha diagnóstica, notificación, tipificación bacteriana, investigación y análisis epidemiológico. Para esto se requieren recursos humanos y técnicos suficientes en varios puntos de esta cadena de vigilancia y control. Por lo tanto, es relevante reflexionar sobre la capacidad de respuesta que dispone el país a comienzos del siglo XXI. Para responder esta pregunta es necesario averiguar cómo funcionan los componentes de esta cadena y así permitir obtener conclusiones. Estos componentes se revisan a continuación. 


\section{Sospecha diagnóstica}

La casi desaparición de la fiebre tifoidea ha hecho que cada vez con menos frecuencia las nuevas generaciones médicas tengan experiencia clínica con estos pacientes y además ha producido una reducción de esta patología en sus estudios curriculares de pregrado. Esto disminuye la sospecha clínica y, en consecuencia, la solicitud de estudios microbiológicos para su diagnóstico. Debido a que el diagnóstico definitivo se obtiene fundamentalmente mediante hemocultivos, la falta sistemática de este recurso en las consultas de atención primaria en el subsistema público de salud (> 60\% de la población) y la tendencia a usar antimicrobianos en cuadros febriles prolongados en forma empírica, hace más difícil que en caso de plantearse la sospecha, el diagnóstico pueda ser documentado. Esta falencia tampoco ha sido resuelta por la incorporación de pruebas diagnósticas rápidas basadas en la detección de anticuerpos como el Typhidot-M®, que ha demostrado un rendimiento competitivo frente a los hemocultivos ${ }^{9}$. En el caso del subsistema privado, este recurso está disponible pero depende críticamente de la sospecha del médico tratante. Ambos factores apuntan a un probable sub-diagnóstico crónico de esta enfermedad, los que no obstante han participado en forma continua durante todo el período en que han declinado las tasas de fiebre tifoidea y no cuestionan la disminución objetiva de esta enfermedad. Debido a que la fiebre tifoidea está ligada a la pobreza y subdesarrollo, es en el subsistema público de salud donde la falta de recursos diagnósticos en atención primaria, tales como la disponibilidad de hemocultivos, genera más problemas con la identificación de la enfermedad, lo que es probablemente enmascarado a su vez por el inicio empírico de antimicrobianos potencialmente activos contra la fiebre tifoidea.

\section{Notificación}

El sistema de notificación de enfermedades infecciosas transmisibles se ha fortalecido en los últimos años hacia el nivel central y se ha eliminado la modalidad de notificación semanal quedando restringida a una notificación de tipo inmediata o diaria. Además, la omisión de este acto administrativo está ligada a sanciones locales. Una serie de agentes bacterianos, incluyendo Salmonella spp., son objeto de vigilancia de laboratorio según el decreto supremo № 158 y deben ser derivados desde los laboratorios clínicos hacia el ISP una vez a la semana para su tipificación, acto que permite contar en forma más rutinaria con aislados para estudios epidemiológicos moleculares. De esta manera, no parece ser el sistema de notificación un aspecto deficiente en el problema bajo análisis. No obstante, es probable que exista un grado de dispersión de este cumplimiento a nivel lo largo del país.

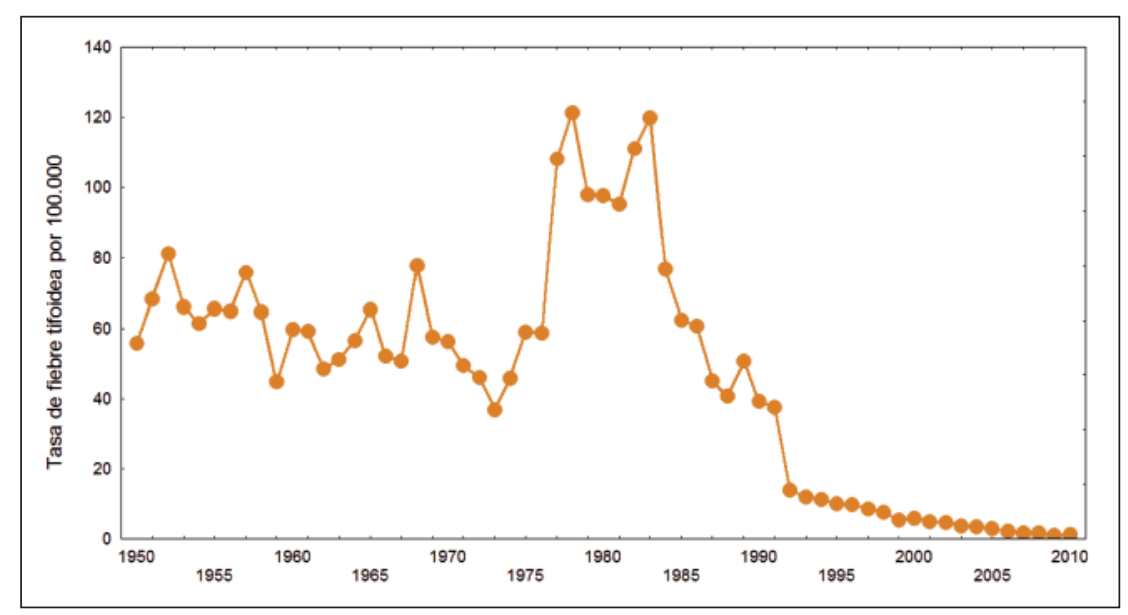

Figura 1. Tasas de fiebre tifoidea en Chile, 1950-2010 (Fuente: Departamento de Epidemiología y Departamento de Estadísticas e Información de Salud del Ministerio de Salud de Chile).

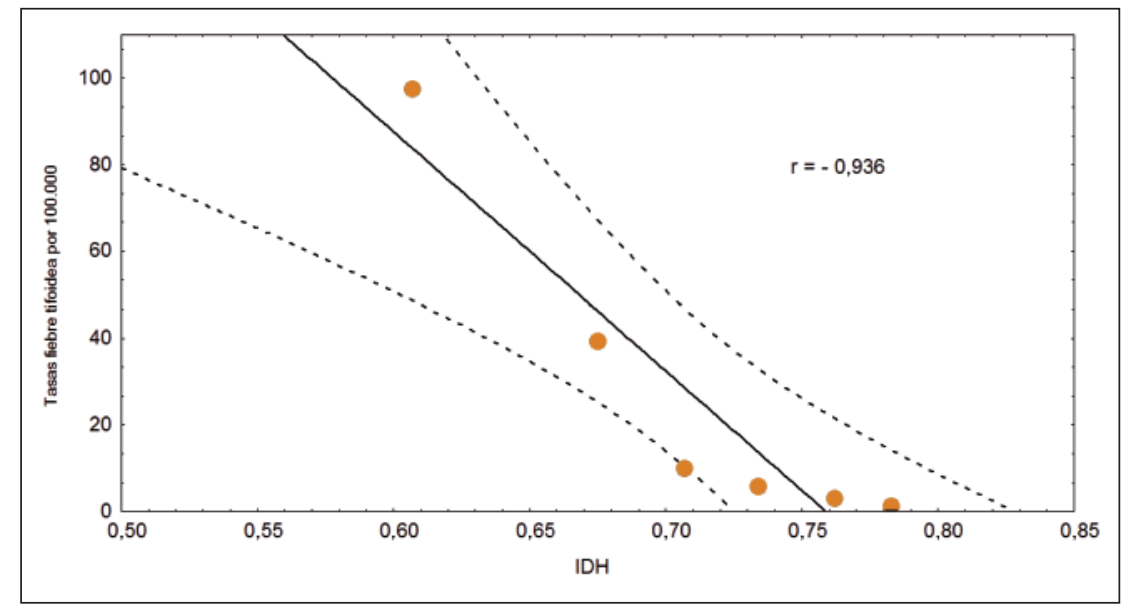

Figura 2. Reducción de tasas de fiebre tifoidea en Chile según avances en IDH para el período 19802010 (Fuente: Departamento de Epidemiología, Departamento de Estadísticas e Información de Salud del Ministerio de Salud de Chile y Programa de las Naciones Unidas para el Desarrollo).

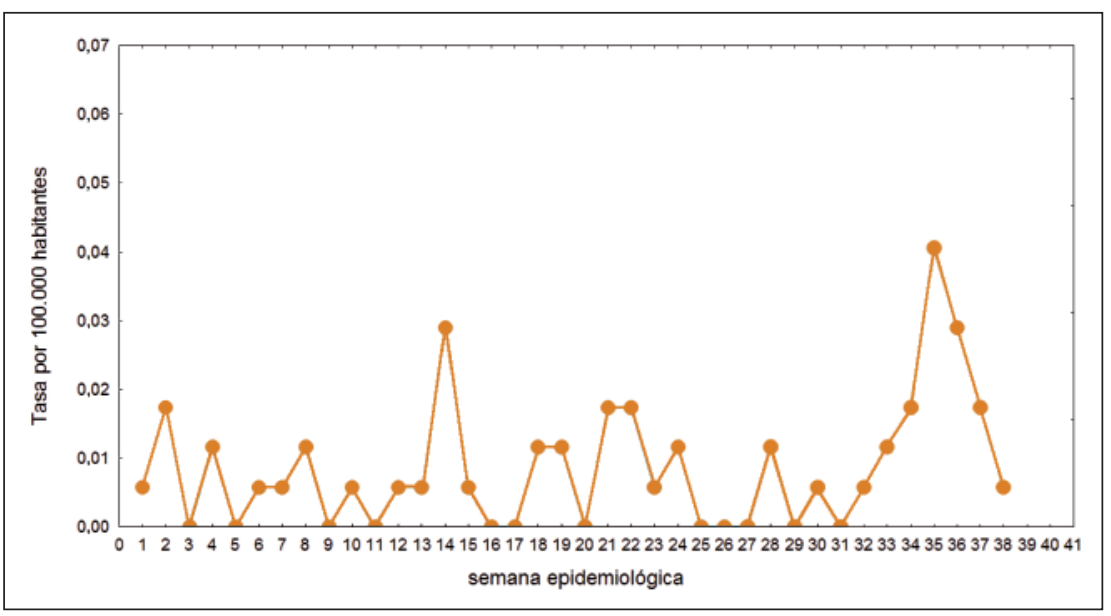

Figura 3. Tasas de fiebre tifoidea por semana epidemiológica, año 2011 hasta semana epidemiológica 38, Chile. Fuente: Instituto de Salud Pública de Chile. 


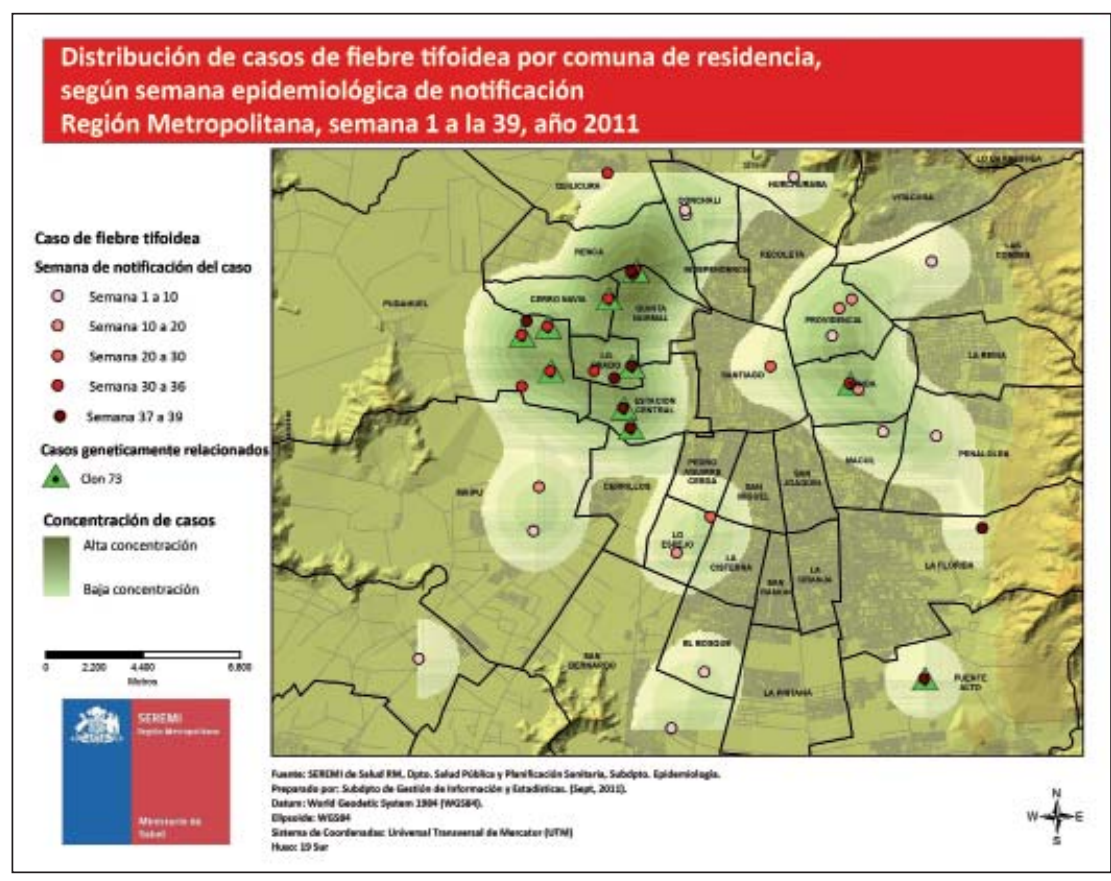

Figura 4. Fuente: ISP de Chile. los recursos humanos integrantes del órgano ejecutor de la fiscalización alimentaria y vigilancia epidemiológica en Chile (llámese SEREMI-Salud), tienen la misma o menor dotación que hace décadas a pesar del aumento de la complejidad de los procesos productivos y de un aumento en 52\% del total de la población en Chile entre 1980 y el 2010 y en 32\% en la RM en los últimos 10 años ${ }^{5}$. Ante este escenario de magros recursos humanos para la fiscalización alimentaria, vigilancia y control, las SEREMI de Salud han restringido su rol fiscalizador rutinario sobre algunos procesos de la industria de los alimentos y los puntos de venta de ellos. Las actividades se concentran en la autorización sanitaria para nuevos puestos de venta de alimentos y en la fiscalización preventiva antes de grandes eventos religiosos o festivos nacionales, pero no en un programa acorde al tamaño poblacional. Las actividades de fiscalización son importantes en el riesgo de fiebre tifoidea ya que, a pesar de la existencia de portadores crónicos de este agente, se minimizan las posibilidades de que este patógeno alcance nuevos hospederos susceptibles. En la Tabla 1 se puede observar que el valor promedio de visitas de fiscalización alimentaria fue de 1.440 actividades cada 100.000 habitantes el año 2010 . Sin embargo, este valor presenta una enorme dispersión que va desde 95 acciones en la RM a 3.124 por cada 100.000 habitantes en Aysén. Las regiones con menos actividades de fiscalización corresponden a la RM (95 por 100.000), Araucanía (234 por 100.000), Coquimbo (811 por 100.000) y Valparaíso (872 por 100.000) (datos construidos con información obtenida del sitio web de las Secretarías Regionales Ministeriales de Salud y de las proyecciones de población del INE).

Como una forma de ejemplificar estas limitaciones, se puede mencionar que la fiscalización de la forma de regadío de cultivo de hortalizas que se consumen crudas y que crecen a ras de suelo en la RM, se ha reducido al mínimo en los últimos años. Esta actividad fue muy importante en los años 90 para confiscar hortalizas que eran regadas con aguas de canales y no de pozos, ya que las primeras tenían una alta concentración de coliformes fecales (> $1.000 \mathrm{ufc} / 100 \mathrm{~mL}$ ). Si se analizan a su vez, los dos últimos años de estas actividades (Tabla 2), se puede concluir que a pesar de que las actividades de fiscalización sobre alimentos se expandieron en $20 \%$ entre el 2009 y el 2010, fue posible observar que en este mismo espacio de tiempo grandes variaciones en el número de actividades analizadas. Al menos cinco regiones redujeron sus actividades el 2010 respecto al 2009 (Arica-Parinacota, Antofagasta, Maule, Bío-Bío, Araucanía y Aysén). El terremoto de febrero del 2010 no puede ser inculpado totalmente por este hecho ya que la disminución también fue observada en zonas no afectadas. En todo el año 2009, oficialmente sólo hubo 107 actividades de control sobre el rubro alimentos en la RM, lo que probablemente 
representa un error y nos impide conocer la dimensión real de estas fiscalizaciones antes del 2010.

Estos datos oficiales indican que las actividades de fiscalización sobre alimentos en Chile no parecen estar aumentando sistemáticamente, no están adaptadas al tamaño poblacional y no parecen siempre orientadas hacia donde ocurre el consumo sino hacia el lugar de la producción agropecuaria o sitios de importación-exportación.

De esta manera, el brazo crítico ante nuevos brotes de fiebre tifoidea parece estar centralizado en la limitada capacidad que tienen los escasos recursos humanos para fiscalizar los alimentos y/o para efectuar estudios de campo que permitan identificar las fuentes de infección desde reservorios humanos. El mejor esfuerzo de los otros componentes de esta cadena, no dará resultados tangibles si no se mejora la cobertura en el personal de los SEREMI de Salud y ello indudablemente está asociado a decisiones políticas presupuestarias de largo plazo y al tamaño que debe tener el Estado. El que estos recursos funcionen adecuadamente es fundamental para establecer un control sobre este riesgo. El no resolverlo mantendrá probablemente la existencia de brotes de mayor o menor cuantía en el corto y mediano plazo.

\section{Salmonella serotipo Enteritidis}

El caso de $S$. Enteritidis es diferente al de fiebre tifoidea en muchos aspectos. Su presentación clínica está centrada en un cuadro autolimitado de diarrea y fiebre que rara vez se acompaña de bacteriemia. El eje diagnóstico está basado en el coprocultivo y los antimicrobianos no acortan su evolución clínica. En Chile, los cuadros clínicos se asocian al consumo de huevos crudos o mal cocidos y productos derivados como mayonesas y merengues, entre otros ${ }^{13}$. La contaminación de estos alimentos se produce en los planteles avícolas por transmisión cruzada, hacinamiento en las jaulas, alimentos para aves sin certificación microbiológica, malas condiciones higiénicas, ausencia de programas de prevención o cadenas de frío en el acopio y transporte, entre otros factores. Hacia fines de los 90, cerca de $6 \%$ de la carne de ave y $0,1 \%$ de los huevos en venta en la RM, estaban contaminados por $S$. Enteritidis ${ }^{10}$. A pesar del bajo porcentaje de contaminación de los huevos, este hecho tiene mayor relevancia epidemiológica debido a su consumo semicrudo ocasional y al enorme volumen de unidades que se consume cada día. La contaminación puede ser transovárica y la indemnidad exterior o limpieza del huevo no asegura su inocuidad. Estudios nacionales demostraron la emergencia de este patógeno en forma reciente y su asociación con la industrialización y concentración de la producción avícola en Chile ${ }^{6,10,11,14}$. La relación con la industrialización se explica por las nuevas formas de concentrar y alimentar las aves, que incluyen productos no naturales en su alimentación. A diferencia de S. Typhi,

\begin{tabular}{|c|c|c|c|}
\hline Región & $\begin{array}{l}\text { Actividades de } \\
\text { fiscalización }\end{array}$ & $\begin{array}{l}\text { Población } \\
\text { estimada }\end{array}$ & $\begin{array}{l}\text { Actividades } \\
\text { por } 100.000 \\
\text { habitantes }\end{array}$ \\
\hline Metropolitana & 6.559 & 6.883 .563 & 95,3 \\
\hline Arica-Parinacota/Tarapacá* & 4.369 & 314.534 & $1.389,0$ \\
\hline Antofagasta & 5.123 & 575.268 & 890,5 \\
\hline Atacama & 2.719 & 280.543 & 969,2 \\
\hline Coquimbo & 5.834 & 718.717 & 811,7 \\
\hline Valparaíso & 15.345 & 1.759 .167 & 872,3 \\
\hline Del Libertador B. O'Higgins & 8.082 & 883.368 & 914,9 \\
\hline Maule & 11.193 & 1.007 .831 & $1.110,6$ \\
\hline Bío-Bío & 34.718 & 2.036 .443 & $1.704,8$ \\
\hline Araucanía & 2.277 & 970.419 & 234,6 \\
\hline De Los Ríos-De Los Lagos* & 22.072 & 836.256 & $2.639,4$ \\
\hline Aysén & 3.276 & 104.843 & $3.124,7$ \\
\hline Total Chile & 127.870 & 17.094 .275 & 748,0 \\
\hline \multicolumn{4}{|c|}{$\begin{array}{l}\text { *Las actividades de las regiones de Arica-Parinacota fueron fusionadas con las de Tarapacá para poder } \\
\text { efectuar el cálculo por unidad de habitantes y lo mismo se hizo para la región de Los Ríos con la de } \\
\text { Los lagos. }\end{array}$} \\
\hline
\end{tabular}

Tabla 2. Actividades de fiscalización de las SEREMI de Salud sobre alimentos reportadas para los años 2009 y 2010.

Fuente: Secretarías Regionales Ministeriales de Salud, Chile

\begin{tabular}{lccc|} 
Región & $\mathbf{2 0 0 9}$ & $\mathbf{2 0 1 0}$ & $\begin{array}{c}\text { Índice actividad sobre alimentos } \\
\mathbf{2 0 0 9}=\mathbf{1 0 0}\end{array}$ \\
Metropolitana & 107 & 6.559 & $6029,91 \%$ \\
Arica- Parinacota & 1.079 & 932 & $-13,62 \%$ \\
\hline Tarapacá & 2.559 & 3.437 & $34,31 \%$ \\
\hline Antofagasta & 5.249 & 5.123 & $-2,40 \%$ \\
\hline Atacama & 817 & 2.719 & $232,80 \%$ \\
\hline Coquimbo & 4.498 & 5.834 & $29,70 \%$ \\
\hline Valparaíso & 10.021 & 15.345 & $53,13 \%$ \\
\hline Del Libertador B. O'Higgins & 4.291 & 8.082 & $88,35 \%$ \\
\hline Maule & 13.266 & 11.193 & $-15,63 \%$ \\
\hline Bío-Bío & 34.929 & 34.718 & $-0,60 \%$ \\
\hline Araucanía & 2.856 & 2.277 & $-20,27 \%$ \\
\hline De Los Ríos & 3.459 & 3.924 & $13,44 \%$ \\
\hline De Los Lagos & 14.949 & 18.148 & $21,40 \%$ \\
\hline Aysén & 3.644 & 3.276 & $-10,10 \%$ \\
\hline Magallanes & 4.838 & 6.303 & $30,28 \%$ \\
\hline Total Chile & 279.156 & 309.675 & $20,00 \%$ \\
\hline
\end{tabular}




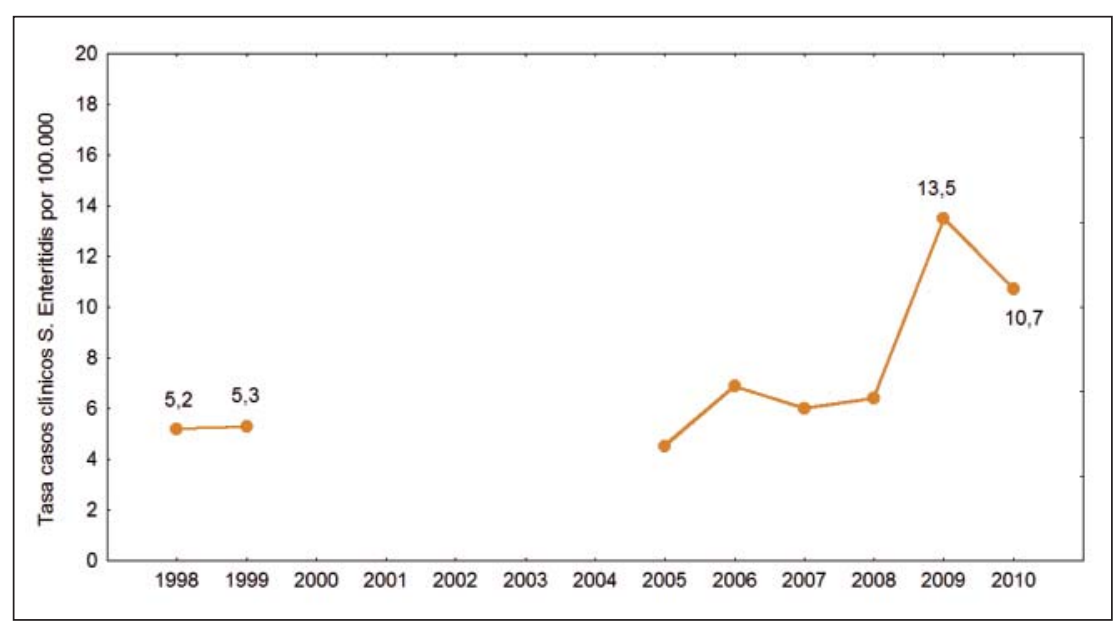

Figura 5. Tasa de casos clínicos de infecciones asociadas a Salmonella serotipo Enteritidis en Chile 1998-1999 y 2005-2010 (sin datos disponibles para años 2000-2004). Fuente: Departamento de Epidemiología del MINSAL y proyecciones de población del INE.

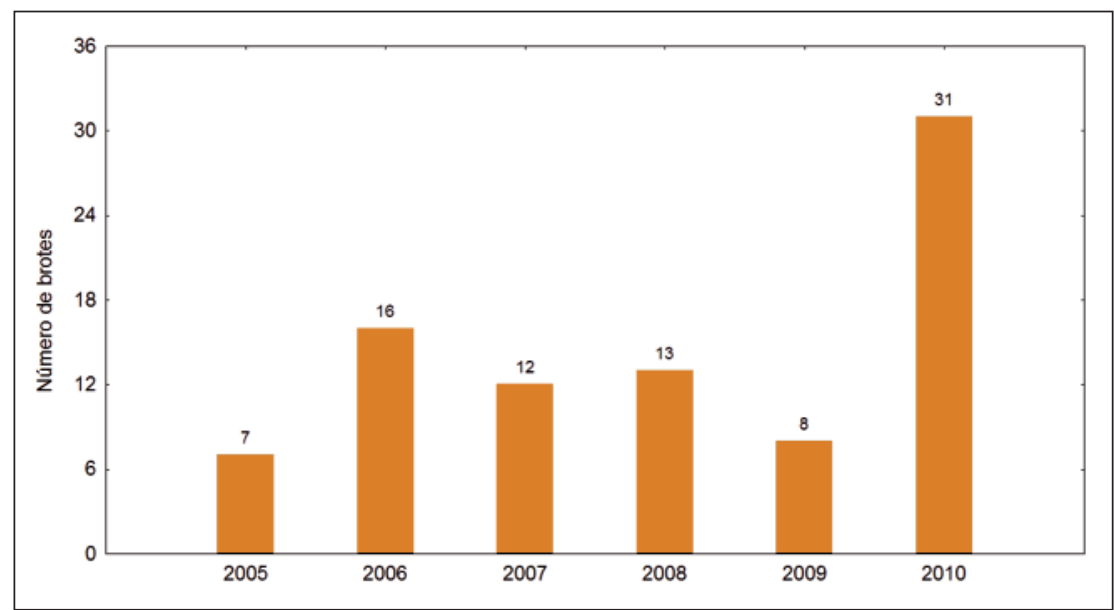

Figura 6. Brotes de toxi-infección alimentaria con participación de Salmonella serotipo Enteritidis reportados en Chile, 2005-2010. Fuente: Departamento de Epidemiología, Ministerio de Salud.

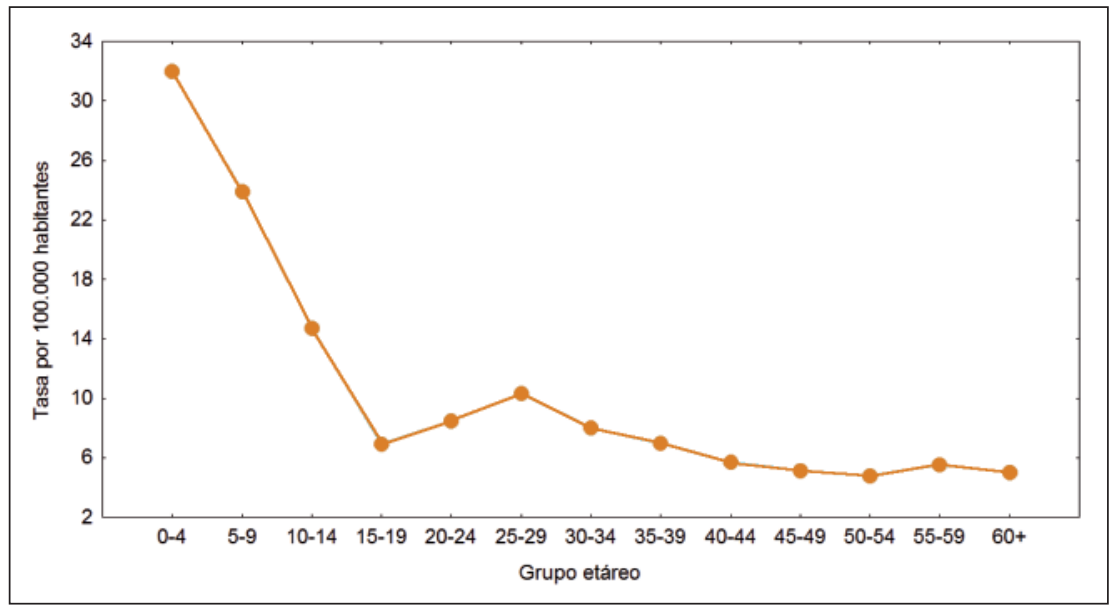

Figura 7. Tasa de incidencia específica por edad de infecciones clínicas por Salmonella serotipo Enteritidis durante el año 2010. Fuente: Departamento de Epidemiología, Ministerio de Salud y proyecciones de población, INE. en este caso no hay un reservorio humano significativo en su cadena de transmisión. ${ }^{12}$ Hacia el año 1999 la tasa de infección por $S$. Enteritidis registrada en el ISP era de 5,3 casos por 100.000 habitantes. Con los datos presentados por el ISP para el año 2010 (1.826 casos), se puede estimar una tasa de 10,7 casos por 100.000 habitantes para ese año, lo que significa que actualmente el problema es el doble que hace una década (Figura 5). Los brotes registrados en Chile asociados a $S$. Enteritidis revelan un aumento importante para los años 2009-2010 (Figura 6). La incidencia específica por edad revela una concentración en niños y adultos jóvenes, hecho compatible con el consumo de productos derivados avícolas específicos (Figura 7).

¿Qué ha permitido entonces que este problema no decline e incluso aumente? En el caso de este patógeno, son los programas de prevención alimentaria en la población y la producción, transporte y venta de productos avícolas derivados en forma segura, los que permiten el control y reducción de este problema. Todos estos componentes en Chile son inexistentes, fragmentarios o inadecuadamente fiscalizados como se analiza a continuación.

\section{Planteles avícola}

Son controlados por el Servicio Agrícola y Ganadero, cuyo fin es fomentar la producción de huevos y de carne de ave. A partir del año 2010 se ha implementado en conjunto con el MINSAL, y la Asociación de Productores de Huevos un Programa Nacional de Control de Salmonella en Aves de Postura, en planteles con alta producción de huevos, que abarque toda la cadena de producción para lograr bioseguridad en planteles, en el empaque, en la fábrica de alimentos, etc. Sin embargo, se desconoce en forma pública, los resultados de los programas de supervisión del SAG sobre las diferentes normativas vigentes de bioseguridad en planteles avícolas desde el 2006, a pesar de que existen indicadores específicos de cumplimiento elaborados por este organismo gubernamental.

La aplicación de programas amplios de control sobre planteles en E.U.A., ha permitido reducir notoriamente las tasas de infecciones clínicas por $S$. Enteritidis en ese país desde el año 1996 en adelante. Las tasas de casos confirmados se redujeron desde 3,9 por 100.000 el año 1994 a cifras inferiores a 2 por $100.000^{13}$. La declinación se ha observado tanto en la frecuencia y magnitud de los brotes como en los casos esporádicos. Las cifras que motivaron una intervención regulatoria y educacional amplia en ese país se iniciaron con valores bastante inferiores a los observados actualmente en Chile, lo que revela nuestras limitaciones para generar una respuesta transversal a este problema.

\section{Control de puntos críticos}

El reglamento sanitario establece en su artículo 69 que los establecimientos de producción, elaboración, 
preservación y envase de alimentos, deberán implementar las metodologías de Análisis de Peligros y Puntos Críticos de Control (HACCP), en toda su línea de producción, conforme lo establecido en la Norma Chilena Oficial NCh 2861 del año 2004. Hacia marzo del 2012, todo tipo de empresa de este tipo debería haber implementado este sistema y ya debería haberse aplicado en los alimentos de mayor riesgo, incluyendo lácteos, huevos y carnes. Actualmente se está considerando postergar los plazos para el cumplimiento de esta norma debido al gran atraso que tiene, especialmente sobre la mediana y pequeña industria. Por otra parte, la supervisión del cumplimiento de esta norma moderna queda sujeta a auditores internos, terceros y al Estado. Sin embargo, la normativa no establece si las desviaciones encontradas bajo este sistema deben ser reportados a la autoridad sanitaria (SEREMI de Salud) ni establece la frecuencia con que el Estado debe efectuar tales controles ya que sólo habla de vigilancia programada que, como vimos, experimenta variaciones en el corto plazo. Es posible entonces, que de acuerdo al crecimiento de la industria de los alimentos en Chile y al pequeño tamaño de los recursos de las SEREMI de Salud, no se pueda cumplir el espíritu de esta normativa aún por bastante tiempo. Actualmente, las SEREMI de Salud por su escasez de recursos, privilegian las visitas o control de puntos críticos a fábricas de alto riesgo como las plantas elaboradoras de cecinas, pasteurizadoras de leche y de alimentos procesados.

\section{Venta de huevos no refrigerados}

A diferencia de los países desarrollados, no se exige en Chile el acopio inicial, transporte y venta de huevos en forma refrigerada para inhibir el crecimiento de un inóculo potencial de Salmonella Enteritidis al interior del huevo ${ }^{8}$. Esta medida ha sido adoptada por países desarrollados desde hace muchos años.

\section{Mayonesa elaborada con huevos frescos}

Sólo a raíz de dos brotes comunitarios y con una persona fallecida durante el año 2011 en la RM, se estableció por primera vez la prohibición de la venta de mayonesa de confección artesanal en los locales de venta de alimentos en Chile, como así mismo en los aderezos que incorporen mayonesa artesanal, mediante la modificación respectiva del Decreto No 977 de 1996, Reglamento Sanitario de los Alimentos de Chile. Este avance importante está vigente desde fines de octubre del 2011. Cerca de la mitad de los brotes reportados han estado asociados a consumo de mayonesa elaborada en forma artesanal o casera ${ }^{4}$ y ello podría indicar que si se fiscalizara intensamente este aspecto y se difundiera su prohibición, podría haber un impacto para reducir el número de casos en Chile.

\section{Educación de la población}

Los programas de educación sanitaria de la población son de baja penetración y duración y muchas veces reactivos al problema contingente. Son costosos y deben ocupar diferentes plataformas y redes sociales lo que limita su alcance. Además, los conceptos en seguridad alimentaria e higiene no forman parte de los contenidos curriculares mínimos actuales en Educación Básica o Media.

Todos estos problemas son agravados por las deficiencias en la fiscalización alimentaria, la capacidad de respuesta y análisis epidemiológico comentados para el caso de la fiebre tifoidea, que como señalamos están probablemente asociados a la baja dotación de recursos humanos de las instituciones encargadas.

\section{Conclusiones}

Los hechos presentados indican que en Chile existen actualmente dos tipos de infecciones por diferentes serotipos de Salmonella con diferente comportamiento epidemiológico, reservorio, manifestaciones clínicas y formas de control. Aunque las tasas de fiebre tifoidea han declinado, el brote reciente demuestra cómo el control ejercido por el desarrollo sanitario y humano en Chile ha fallado para impedir completamente la circulación de este agente. Por otra parte, se observa cómo las infecciones zoonóticas por Salmonella Enteritidis han aumentado en Chile y revelan la falta de un programa transversal de la sociedad para tratar este problema. El Programa Nacional para el Control de Salmonelosis en aves, asociado a la nueva reglamentación sobre la prohibición de elaborar mayonesas artesanales en locales públicos, no tendrá el impacto esperado si no va acompañado de un fuerte programa educacional a nuestra población y además de un importante programa de fiscalización a esos locales por parte de la Autoridad Sanitaria de cada región del país. En ambas salmonelosis, se puede observar cómo los sistemas de notificación y tipificación bacteriana están trabajando a tiempo real y han facilitado el análisis epidemiológico del problema. Sin embargo, también es posible observar que la capacidad de fiscalización alimentaria y estudio epidemiológico que ejercen los órganos de gobierno está seriamente limitada por la falta de inversión crónica en capital humano. Es urgente resolver estas limitaciones a través de una discusión pública sobre el tamaño y rol que debe tener el Estado en el tema de la seguridad alimentaria y en sus entes fiscalizadores. Los países desarrollados han creado sistemas integrados de vigilancia de las enfermedades entéricas, tales como el European Food and Waterborne Diseases and Zoonoses Surveillance Network de la Comunidad Económica Europea. (sitio web http://ecdc.europa.eu/en/activities/surveillance/ european_surveillance_networks/Pages/european_survei- 
llance_networks.aspx). Este mismo enfoque se aplica en Estados Unidos mediante el Centers for Disease Control and Prevention a través de la Division of Foodborne, Waterborne and Environmental Diseases (sitio web http:// www.cdc.gov/ncidod/dbmd/phlisdata/salmonella.htm.) Estos programas están dedicados a monitorizar tendencias pero, especialmente, a generar un análisis y respuesta rápida ante amenazas a la salud pública en las llamadas actividades de inteligencia epidemiológica. Actualmente, Chile tiene el gran desafío de incrementar sus actividades de supervisión, vigilancia, análisis y respuesta rápida en caso de infecciones de transmisión entérica.

\section{Resumen}

Durante el año 2011 dos brotes de infecciones por Salmonella afectaron la Región Metropolitana (RM) de Chile: uno de fiebre tifoidea y otro de gastroenteritis por Salmonella serotipo Enteritidis, infecciones que habían declinado en los últimos años. Se analizan las probables causas de esta reemergencia. Para el desarrollo de este trabajo se consultaron sitios web gubernamentales y de las Naciones Unidas. Las tasas de fiebre tifoidea han declinado progresivamente hasta tasas muy bajas siendo la actual cercana a un caso por 100.000 habitantes, disminución asociada a las mejorías en las condiciones de vida de la población que se expresa por el índice de desarrollo humano alcanzado. El brote del año 2011 estuvo asociado a un clon predominante que afectó al sector occidente de la RM y donde el único factor de riesgo involucrado fue el consumo de verduras adquiridas en distintas ferias libres del mismo sector, sin lograr la identificación del sitio de origen. Aunque la notificación de esta enfermedad se ha optimizado en los últimos años, al igual que la capacidad de tipificación molecular por parte del Laboratorio de Referencia (ISP), este brote se produce en forma coincidente con un bajo número de fiscalizaciones alimentarias en la RM (la más baja de Chile), explicado probablemente por el bajo número de personal en la repartición responsable en relación al tamaño de la población. En el caso de las infecciones por Salmonella serotipo Enteritidis, la tasa actual es el doble de la observada hace una década (10,7 vs 5,3 casos por 100.000 habitantes) con un incremento progresivo de los brotes alimentarios ligados a este microorganismo desde el 2005 (7 a 31 brotes anuales). La persistencia de este problema está asociada a una escasa actividad fiscalizadora del Estado de Chile hacia los planteles avícolas, a la ausencia de una cadena de frío en la distribución y venta de huevos, y a la falta de educación sobre este tema en la población. La reciente normativa de prohibir la venta de mayonesa elaborada artesanalmente, es un paso importante cuyo impacto aún debe ser evaluado. Conclusiones: La persistencia y reemergencia de ambas salmonelosis en Chile sugiere la existencia de problemas crónicos sobre el tamaño y capacidad del Estado en la seguridad alimentaria del país.

\section{Referencias}

1.- Contreras P. Detectan brote de fiebre tifoidea en sector occidente de Santiago. Diario La Tercera. Edición 13 de septiembre 2011. Disponible en: http://latercera.com/noticia/ nacional/2011/09/680-392576-9-detectanbrote-de-fiebre-tifoidea-en-sector-occidente-desantiago.shtm

2.- Anónimo. Se confirma presencia de Salmonella en brote de gastroenteritis en Peñalolén. Diario El Mercurio. Edición 29 de agosto del 2011. Disponible en: http://www.emol. com/noticias/nacional/2011/08/29/500344/seconfirma-presencia-de-salmonella-en-brote-degastroenteritis-en-penalolen.html

3.- Solari V. Situación epidemiológica fiebre tifoidea y paratifoidea Región Metropolitana 2011 semana 39 (al 30 de septiembre) Disponible en: http://www.ispch.cl/sites/ default/files/Situacion_Epi_Fiebre_tifoidea_ Paratifoidea\%20RM_39\%20.pdf

4.- Departamento de Epidemiología Ministerio de Salud de Chile. Informe Salmonella Enteritidis. Disponible en: http://epi.minsal.cl/ epi/html/bolets/reportes/Salmonella/Informe_
Salmonella_2011.pdf

5.- Instituto Nacional de Estadísticas. Chile: Proyecciones y estimaciones de población. 1990-2020. País y Regiones. Disponible en http://palma.ine.cl/demografia/menu/ EstadisticasDemograficas/DEMOGRAFIA.pdf

6.- Fica A, Alexandre M, Prat S, Fernández A, Fernández J, Heitmann I. Cambios epidemiológicos de las salmonelosis en Chile. Desde Salmonella Typhi a Salmonella Enteritidis. Rev Chilena Infectol 2001; 18: 85-93.

7.- $\quad$ Programa de las Naciones Unidas para el Desarrollo. Tendencias del Índice de Desarrollo Humano 1980-2010. Disponible en: http://hdr. undp.org/en/media/HDR_2010_ES_Table2_ reprint.pdf

8.- MINSAL. Reglamento Sanitario de los Alimentos. DTO. № 977/96. Actualizado Junio 2010. Disponible en: http://www.minsal.gob. cl/portal/url/page/minsalcl/g_proteccion/g_ alimentos/reglamento_sanitario_alimentos.html

9.- WHO. Background document. The diagnosis, treatment, and prevention of typhoid fever. Disponible en: http://whqlibdoc.who.int/ hq/2003/WHO_V\&B_03.07.pdf
10.- Alexandre M, Pozo C, González V, Martínez M C, Prat S, Fernández A, et al. Detección de Salmonella Enteritidis en muestras de productos avícolas de consumo humano en la Región Metropolitana. Rev. Méd. Chile, 2000; 128: 1075-83.

11.- Fernández J, Fica A, Ebensperger G, Calfullán H, Prat S, Fernández A, et al. Molecular epidemiology of Chilean Salmonella Enteritidis isolates by pulsed-field gel electophoresis and bacteriophage typing. J Clin Microbiol 2003; 41: 1617-22.

12.- Hedican E, Hooker C, Jenkins T, Medus C, Jawahir S, Leano Fet al. Restaurant Salmonella Enteritidis outbreak associated with an asymptomatic infected food worker. J Food Prot 2009; 72; 2332-6.

13.- Braden C R. Salmonella enteric serotype Enteritidis and eggs: A national epidemic in the United States. Clin Infect Dis 2006; 43: 512-7.

14.- Prat S, Fernández A, Fica A, Fernández J, Alexandre M, Heitmann I. Tipificación fágica de aislados de Salmonella enteritidis de muestras clínicas, alimentarias y avícolas en Chile. Rev Panam Salud Pública-Pan Am J Public Health 2001; 9: 7-12. 